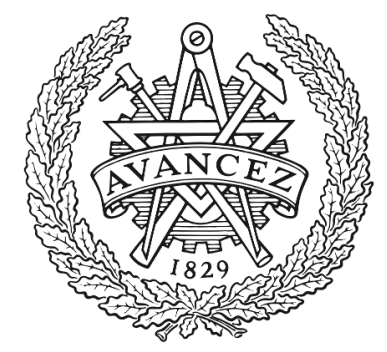

\title{
CHALMERS
}

UNIVERSITY OF TECHNOLOGY

\section{Numerical analysis of a vehicle wake with tapered rear extensions under yaw conditions}

Downloaded from: https://research.chalmers.se, 2023-04-26 14:01 UTC

Citation for the original published paper (version of record):

Urquhart, M., Sebben, S., Sterken, L. (2018). Numerical analysis of a vehicle wake with tapered rear extensions under yaw conditions. Journal of Wind Engineering and Industrial Aerodynamics, 179: 308-318. http://dx.doi.org/10.1016/j.jweia.2018.06.001

N.B. When citing this work, cite the original published paper. 
Numerical analysis of a vehicle wake with tapered rear extensions under yaw conditions

Magnus Urquhart $^{* c}$, Simone Sebben* and Lennert Sterken ${ }^{\dagger}$

${ }^{*}$ Chalmers University of Technology

${ }^{\dagger}$ Volvo Car Corporation

${ }^{c}$ Corresponding author. E-mail address: magnus.urquhart@chalmers.se (M. Urquhart). 


\section{Abstract}

Since recent years governmental legislation and consumer demands are driving the development of more energy efficient road vehicles. One of the aspects considered when increasing efficiency is the aerodynamic performance of the vehicle. The focus of this paper is on side wind effects on drag on a vehicle with tapered rear extensions. For this, numerical simulations are analysed using post-processing techniques such as Proper Orthogonal Decomposition (POD) and Two-point correlation. The extensions protrude $150 \mathrm{~mm}$ from the perimeter of the base and are investigated in two configurations: with a smooth taper and with an added kick, which realigns the perimeter base flow to the vehicle's driving direction.

As the incoming flow angle is increased drag increases for the investigated angles. The smooth extensions provide the greatest drag improvement at $0^{\circ}$-yaw while the extensions with a kick yield addition benefit at yaw, effectively reducing the vehicles drag sensitivity to side wind. A large scale twisting motion is present at yaw for the baseline and smooth extensions configurations which is reduced when adding a kick to the extension.

\section{Keywords}

Tapered extensions, Yaw, Drag, Unsteady, Wake, POD

\section{Introduction}

As part of the global efforts towards a sustainable society, OEMs are working on solutions to decrease emissions and increase the energy efficiency of road vehicles. Stricter emission targets, electric vehicle range and increased consumer demand are all driving factors in this development. The dominant source of resistance for a vehicle driving on a flat road at speeds above $80 \mathrm{~km} / \mathrm{h}$ is the aerodynamic drag [1]. Reducing aerodynamic drag has become increasingly important with increasing road speeds and regenerative braking. This is reflected in the new standardised driving cycle WLTP which, compared to the previous driving cycle NEDC, puts more emphasis on the energy loss due to aerodynamic drag [2].

Road vehicles are considered bluff bodies, and as such are characterised by massively separated wakes dominated by pressure drag. The pressure drag is largely dependent on the pressure difference between the front and rear end. To reduce the pressure difference, the rear end of road vehicles has been, and continues to be, an area of development and research. Different devices and methods have been tested to alter the wake flow and aid in the pressure recovery. Examples of such devices are: cavities, ventilated cavities, boat tailing flaps, passive bleed, short rear end tapers, vortex generators, synthetic jets, side skirts and blowing techniques $[3-12]$. The focus of this paper is on the wake flow features of a road vehicle with tapered rear end extensions, with and without an additional flow aligning feature referred to as a kick.

In a previous study, using the same vehicle geometry as in this investigation, Sebben et al. [13] found a reduction in the unsteady surface pressure fluctuations when adding the extensions. Other authors have also reported a reduction in the unsteadiness of the wake using similar geometrical modifications [3, 14]. Perry [15] investigated the wake characteristics on a simplified vehicle body for varying top and bottom tapers using particle image velocimetry. The lowest drag was found for a $16^{\circ}$ top taper and a $6^{\circ}$ bottom taper, yielding a balanced wake which impinged near half the base height. In the study several configurations were investigated and it was noted that the velocity magnitude within the wake was larger for the configuration with lowest drag compared to the baseline. Perry et al. investigated the wake closure point, size and the kinetic closed for all configurations. The extensions are placed at the

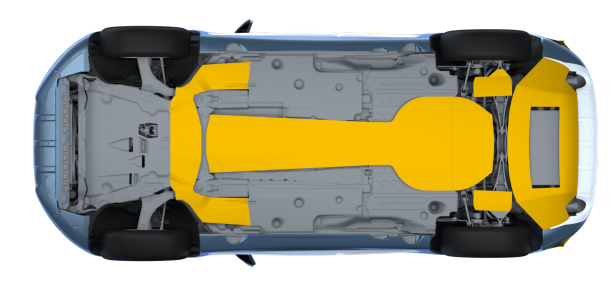

Figure 1: Underbody with additional panels coloured in yellow. C.

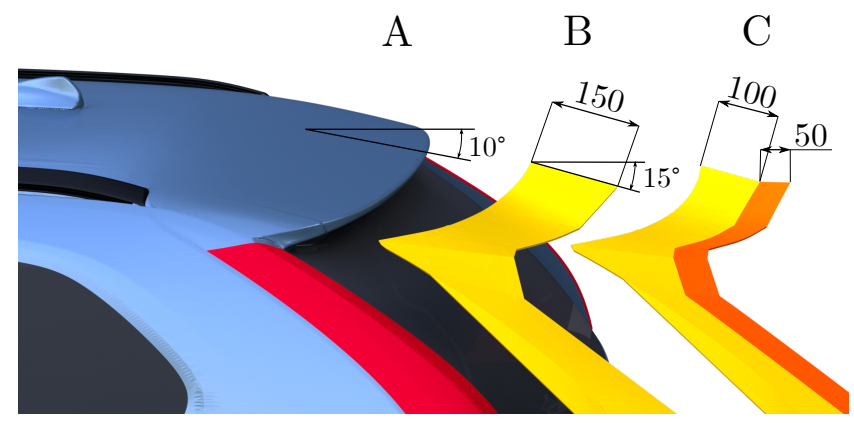

Figure 2: Close-up of the extensions with measurements in [mm]. vature. The taper is increased by approximately $5^{\circ}$ compared to the vehicles original taper of approximately $10^{\circ}$. They are $150 \mathrm{~mm}$ long where the last $50 \mathrm{~mm}$ of the extensions with a kick is pointing in the freestream direction as shown in Figure 2. The extensions cover the top and sides of the vehicle which can be seen in Figure 3. The configurations will heron be called: baseline, $A$, smooth extensions, $B$, and extensions with a kick $C$. energy within the wake; however, no clear connection to drag was found.

This work further investigates the unsteady wake, for a vehicle with extensions, using velocity fields in three wake planes as ell as unsteady surface pressure measurements from numerithe results are analysed in straight and yawed flow conditions using spectral methods and Proper Orthogonal Decomposition (POD). POD is a modal decomposition technue and has been used to investigate fluid flows and extract ent structures $[13,16]$. The investigation is performed for three geometrical configurations: without extensions, referenced to as the baseline $(A)$; with smooth tapered extensions $B)$ and tapered extensions with kick, $(C)$. All three geometrepresenting a continuous side wind condition.

\section{Methodology}

The geometry and the numerical procedure including post processing techniques are described in this section.

\subsection{Geometry}

The test object used in this study is a modified version of a Volvo XC60 AWD, which has been used in previous studies $13,17,18]$. The full size, fully detailed geometry features additional underbody panels as shown in Figure 1. The additional underbody panels were added to improve the flow quality from under the vehicle to the wake. The upper and lower grille are ase, tangential to the vehicle body following the existing cur-

Roof drop and extension angle are referenced to the horizon. 


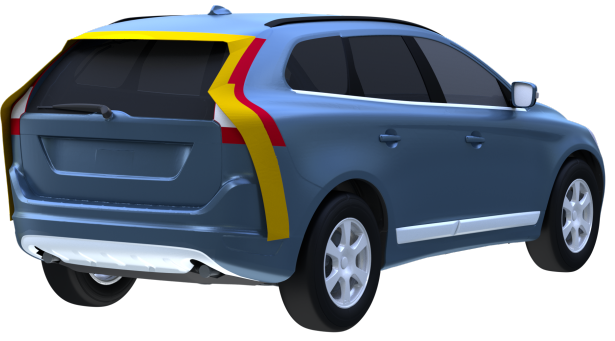

Figure 3: Rear view of the smooth extensions, configuration $B$

\subsection{Numerical set-up}

The numerical simulations were performed using Star-CCM+, which is a Finite Volume Method (FVM) CFD solver. The turbulence is modelled using a hybrid RANS-LES, two equation Improved Delayed Detached Eddy Simulation (IDDES) SST $k-\omega$ turbulence model. A $2^{\text {nd }}$-order temporal and spatial discretization scheme is used where the gradients are computed using a hybrid Gauss-Least Squares method (Hybrid-LSQ). All simulations are run incompressible at $100 \mathrm{~km} / \mathrm{h}$. The resulting Reynolds number is approximately $3 \times 10^{6}$ based on the vehicle length.

The timestep used is $2.5 \times 10^{-4} s$, giving a Courant number of less than 1 in the majority of the domain. The number of inner iterations between timesteps is set to 7 . The convergence within inner iterations was investigated in two points in the wake shear layer were the velocity was monitored. The flow reached convergence for the investigated points where the last 2-3 inner iterations showed little to no change in the monitored values. The timestep size was investigated by reducing it to $1.0 \times 10^{-4} s$, showing less than $0.001 \Delta C_{D}$, or $0.3 \%$ difference in drag between the timesteps.

Each case is comprised of approximately $130 \times 10^{6}$ hexahedral dominant cells where regions such as the wake shear layer, mirrors and underbody are refined. The majority of the car exterior and underbody panels, which are facing the external flow, have 12 prism layers spanning $8 \mathrm{~mm}$ with a first cell height of $0.015 \mathrm{~mm}$ resulting in a $y^{+}<1$ as required by the wall treatment. The resulting growth ratio in the near wall prism is 1.6 which, as investigated by Hobeika et al. [19], was found to be adequate using a similar numerical setup, mesh resolution and Reynolds number. The wheel geometry is smooth and deformed to represent a loaded tire. The wheel rotation is modelled as a rotating wall with Moving Reference Frame (MRF) zones in the spokes, following the same setup as in [13].

The mesh resolution in the separated wake region was evaluated using two-point correlation of the longitudinal velocities. According to Davidson [20], the energy spectra, as well as the resolved turbulent kinetic energy ratio, are poor measures of LES resolution. A better alternative was found to be a twopoint correlation where the number of correlated cells give an indication of the mesh resolution. This will be further explained in section 3.3.3. Two-point correlation for twenty points was
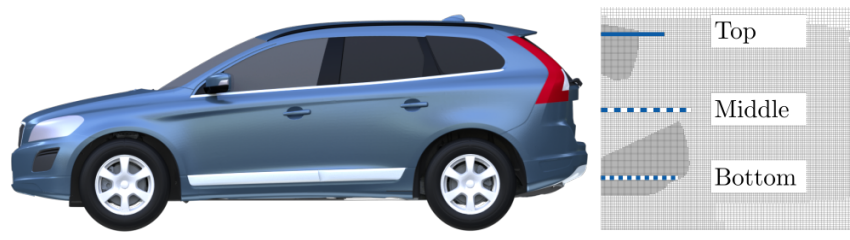

Figure 4: Location of three investigated lines

performed on the centerline plane for the baseline configuration without yaw. The plane and three of the investigated locations can be seen in Figure 4. The reference cell used for the correlation is the cell located closest to the vehicle body for each line. Each investigated point showed similar trends as seen in Figure 5 , with more than twenty cells correlated in the downstream direction. Davidson [20] concluded that coarse LES preferably

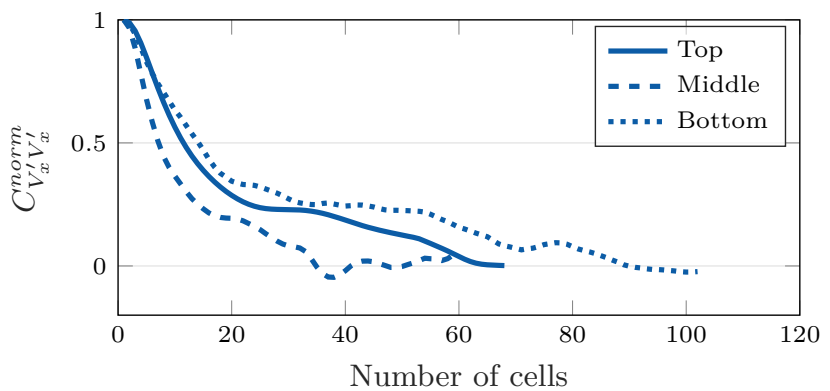

Figure 5: Normalised two-point correlation $100 \mathrm{~mm}$ behind the vehicle

contained more than eighth correlated cells, which was fulfilled for each investigated point. The smallest cells in the wake plane in Figure 4 have and edge length of $6 \mathrm{~mm}$.

The computational domain is made to replicate open road conditions. The dimensions are base on the vehicle length, $l$, with the tunnel being $18.5 l$ long, $4.3 l$ tall and $6.5 l$ wide with the inlet placed $5.5 l$ upstream of the vehicle, see Figure 6 . The left wall of the domain, in the driving direction, is an inlet boundary condition and the right side an outlet boundary condition to simulate side wind.

The velocity magnitude is kept constant for all yaws as to not introduce additional energy to the flow while the driving speed is kept constant at $100 \mathrm{~km} / \mathrm{h}$. Unsteady pressure data was saved

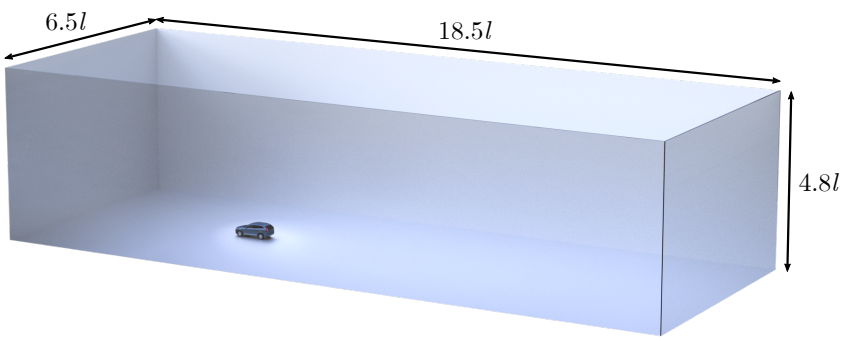

Figure 6: Computational domain

on the base and on three planes in the wake of the vehicle, Figure 7. All planes begin $100 \mathrm{~mm}$ behind the vehicle with the $y$-normal plane positioned on the centerline and the z-normal plane positioned on the halfway point between the roof and underbody. The three velocity components were saved on the wake planes in addition to the pressure. All unsteady data is sampled at $1000 \mathrm{~Hz}$, which is shown to be large enough for converge POD results of the largest modes, see section 3.3.2.

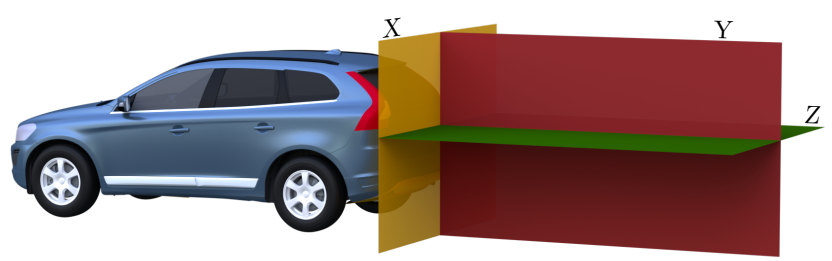

Figure 7: Wake planes where transient data is collected

The flow field and forces were averaged for $3 \mathrm{~s}$ after the flow had developed, resulting in 15 vehicle flow passages. A run- 
ning average was performed for all investigated configurations, showing less than 1 drag count difference to the mean over the last $0.5 s$ when averaging for $3 s$. This was investigated further for the baseline case at $0^{\circ}$-yaw showing the same trend for longer averaging times, see Figure 8 . The results in this work is are presented in terms of counts where 1 drag count is $0.001 C d$. The values in Figure 8 are presented in reference to the mean with the dotted lines indicating the $\pm 1 C_{D}$ counts interval. Even though the disturbances in the force were below

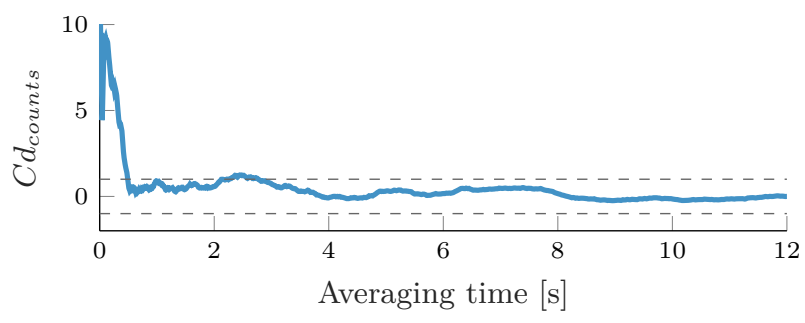

Figure 8: Running average of drag

one count for the last $0.5 \mathrm{~s}$ when averaging for $3 \mathrm{~s}$, longer averaging times were used to gather additional transient data for the $0^{\circ}$ - and $5.0^{\circ}$-yaw cases. The transient data was saved for $12 \mathrm{~s}$ to capture a sufficient amount of low frequency events to be used in the temporal post-processing.

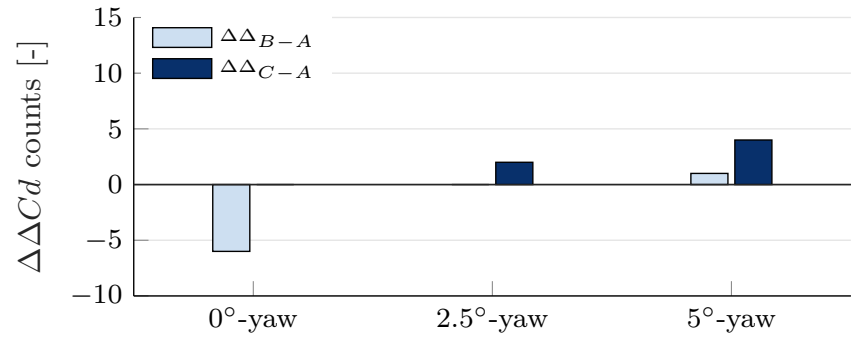

Figure 9: Delta-delta coefficient of drag change. CFD vs wind tunnel.

With this numerical setup, the predicted drag change between configurations is on average within \pm 2 counts of experiments as shown in Figure 9. The predicted drag change difference between CFD and wind tunnel experiments for configuration $B-A, \Delta \Delta_{B-A}$, would be defined as

$$
\Delta \Delta_{B-A}=\left(C d_{B}-C d_{A}\right)_{\mathrm{CFD}}-\left(C d_{B}-C d_{A}\right)_{\text {Test }} .
$$

Additionally, the trend for each configuration or flow condition was predicted correctly. In other words, the sign of the drag change due to yaw or configuration changes, in CFD, consistently matched test results for all investigated configurations. The experimental data is taken from [13].

\subsection{Post-processing}

\subsubsection{Local drag}

The aerodynamic drag of an object can be calculated by applying the momentum equation to a control volume surrounding the object. The control volume should be selected large enough so that only the wake plane is affected by the object and the flow is assumed incompressible [21]. With the aforementioned assumptions, the drag can be expressed as

$$
\begin{aligned}
C_{D} A= & \int_{\Omega}\left[\frac{\partial V_{x}}{\partial t}\right] d \Omega+\int_{S}\left[\left(C_{P^{\text {tot }} \infty}-C_{\text {Ptot }}\right)+\right. \\
& \left.-\left(1-\frac{V_{x}}{V_{\infty}}\right)^{2}+\left(\frac{V_{y}^{2}+V_{z}^{2}}{V_{\infty}^{2}}\right)+\tau_{x x}\right] d S
\end{aligned}
$$

where $\Omega$ and $S$ are the volume and wake surface integral, respectively. Note that this is an instantaneous measure and it's often of interest to look at the time averaged results. By time averaging eq. (2), the volume integral goes to zero. Remembering that the time average of a square term is defined as

$$
\overline{\xi^{2}}=\lim _{T \rightarrow \infty} \frac{1}{T} \int_{T_{0}}^{T_{0}+T} \xi^{2}(t) d t=\bar{\xi}^{2}+\overline{2 \bar{\xi} \bar{\xi}}+\overline{\xi^{\prime 2}}=\bar{\xi}^{2}+\overline{\xi^{\prime 2}}
$$

where $\xi$ is a flow variable such as pressure or velocity, $\xi^{\prime}$ denotes the fluctuating part and $\bar{\xi}$ is the time average. From equation (3) it can be see that each nonlinear term leads to additional terms. The additional terms are usually omitted by assuming steady flow [22]. Neglecting to include the unsteady terms when calculating the drag from a wake plane resulted in an overprediction of drag by as much as $20 \%$ for the vehicle used in this study. Lastly, the viscous term in equation (2), $\tau_{x x}$, is usually omitted as it is small compared to the other terms. In this work, the viscous term is orders of magnitude smaller than any other term and is therefore omitted. The total time-averaged drag can then be expressed in a wake plane as

$$
\begin{aligned}
C_{D} A= & \int_{S} \underbrace{}_{\text {Profile drag }} \underbrace{\left(C_{\text {Ptot } \infty}-C_{\text {Ptot }}\right)-\left(1-\frac{V_{x}}{V_{\infty}}\right)^{2}}_{\text {Crossflow drag }}+ \\
& +\underbrace{\left.\left.\frac{V_{y}^{2}+V_{z}^{2}}{V_{\infty}^{2}}\right)\right] d S}
\end{aligned}
$$

where $C_{P t o t}$ refers to the total pressure coefficient and the subscript $\infty$ denotes the freestream value. Equation 4 can be solved for in a simplified form, without any crossflow terms. By substituting the total pressure with static and dynamic pressure the crossflow trems cancel.

$$
C_{D} A=\int_{S}\left[-C_{P}-2\left(\frac{V_{x}^{2}}{V_{\infty}^{2}}-\frac{V_{x}}{V_{\infty}}\right)\right] d S .
$$

The drag computed by the local drag equation contains a contribution from the ground which in this case was found to be small, approximately $-2 C_{D}$ counts.

The last term in the local drag equation, equation (4), has been commonly referred to as the lift induced- or vortex drag term; however, $\mathrm{Wu}[23]$ recognised that this term is not solely comprised of lift induced- or vortex drag, since the flow contributing to the third term can exist without causing lift or being part of a vortex. Therefore the last term in eq. (4) will heron be called the crossflow drag. The local drag equation has been used to quantitatively and qualitatively investigate road vehicle wakes by several authors, some examples are [6, 18, 24-29]. The local drag equation can be advantageous to use since it's directly related to the drag and allows for visual inspection of the losses in the flow.

\subsubsection{Proper Orthogonal Decomposition}

Proper Orthogonal Decomposition (POD) is a modal decomposition technique which has been used in research to extract information about time varying turbulent flows. A brief description of the technique is introduced here. It's assumed that the flow can be decomposed as

$$
\mathbf{X}(\mathbf{r}, t)=\sum_{i=1}^{m} a_{i}(t) \phi_{i}(\mathbf{r})
$$


where $\phi_{i}(\mathbf{r})$ is the $i$-th POD mode and $a_{i}(t)$ is the mode coefficients containing the temporal information. $\mathbf{X}$ is the space and time dependent flow variables arranged as

$$
\mathbf{X}=\left[\begin{array}{cccc}
x_{1, t_{1}} & x_{1, t_{2}} & \ldots & x_{1, t_{m}} \\
x_{2, t_{1}} & x_{2, t_{2}} & \ldots & x_{2, t_{m}} \\
\vdots & \vdots & \ddots & \vdots \\
x_{n, t_{1}} & x_{n, t_{2}} & \ldots & x_{n, t_{m}}
\end{array}\right]
$$

with $n$ data points and $m$ snapshots. In this work, the matrix $\mathbf{X}$ contains the pressures on the base as well as pressures and velocities in the three wake planes. In all the presented results the POD mode of pressure is presented. The objective of POD is to find the basis vectors that optimally represent the data in a $L^{2}$ sense. In other words, the method seeks to find the best representation of the original data using the least amount of modes. This can be solved by solving the eigenvalue problem

$$
\mathbf{Y} \psi_{i}=\lambda_{i} \psi_{i}, \quad \mathbf{Y}=\mathbf{X X}^{T}
$$

where the modes are calculated as

$$
\phi_{i}=\mathbf{X} \psi_{i} \frac{1}{\sqrt{\lambda_{i}}} .
$$

This is often referred to as the classical POD method. Noting that $\mathbf{X X}^{T} \in \mathbb{R}^{n \times n}$, this method can quickly become prohibitively expensive if the number of cells is large and larger than the number of snapshots. The more common method used in fluid mechanics, where $n>m$, is the method of snapshots which solves the eigenvalue problem

$$
\mathbf{X}^{T} \mathbf{X} \psi_{i}=\lambda_{i} \psi_{i}
$$

which is a smaller eigenvalue problem of size $m \times m$. Note that this formulation assumes the data sampled in $\mathbf{X}$ to be on a equidistant mesh, for this investigation the mesh isn't uniform. The cell volume should be included for non uniform sampling by replacing $\mathbf{X}^{T} \mathbf{X}$ with $\mathbf{X}^{T} \mathbf{W} \mathbf{X}$ where $\mathbf{W}$ is a diagonal matrix containing the cell volume [30]. The temporal coefficients $a_{i}(t)$ are constructed as

$$
a_{i}(t)=\sqrt{\lambda_{i}} \psi_{i}^{T}
$$

In practice this can also be solved using a Singular Value Decomposition (SVD)

$$
\mathbf{X}=\boldsymbol{\Phi} \boldsymbol{\Sigma} \boldsymbol{\Psi}^{T}
$$

with the modes as, $\phi_{i}\left(\mathbf{r}=\Phi=\left[\phi_{1}, \phi_{2}, \ldots \phi_{m}\right] \in \mathbb{R}^{n \times m}\right.$ and the coefficients as $a_{i}(t)=\boldsymbol{\Sigma} \boldsymbol{\Psi}^{T}=\left[a_{1}, a_{2}, \ldots a_{m}\right] \in \mathbb{R}^{m \times m}$. More information about the POD method can be found in [16, 30].

\section{Mode convergence}

In order to determine if a sufficient amount of transient events are contained in the snapshot matrix $\mathbf{X}$, the snapshot mode convergence was investigated. Muld et al. [30] suggest two metrics for investigating mode convergence, the orthogonality between modes and the $L^{2}$-norm, with both methods giving good results. Here the $L^{2}$-norm is used to investigate the convergence of modes, and is defined as

$$
L^{2}=\min \left(\left\|\phi_{i, t}-\phi_{i, e n d}\right\|,\left\|\phi_{i, t}-\left(-\phi_{i, e n d}\right)\right\|\right)
$$

for the $i$-th modes. Each mode is normalised to length 1 before the comparison. $\phi_{i, \text { end }}$ is the $i$-th mode used for comparison, containing the snapshots for all $12 \mathrm{~s}$. The minimum is used since the sign of each mode is not known. The mode is considered converged if the $L^{2}$-norm approaches 0 before the last snapshot (the last snapshot is by definition 0 ). This means that adding additional snapshots does not change or add information to the

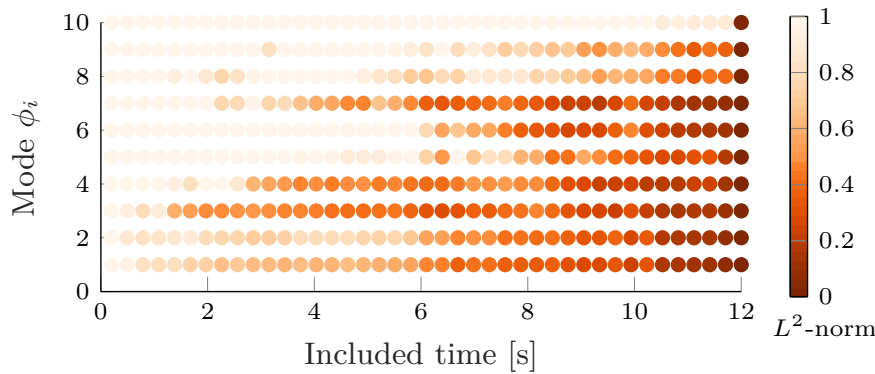

Figure 10: Snapshot mode convergence for the first ten modes.

modal decomposition. The snapshot convergence for the first 10 modes are presented in Figure 10, for the baseline geometry at $0^{\circ}$ yaw. Figure 10 indicates that the higher energy modes are converged after $11 \mathrm{~s}$.

In addition to the snapshot mode convergence rate, the sampling rate convergence was also investigated. The sampling rate convergence is computed the same way as for the snapshots, with the difference being the modes $\phi_{i, t}$ now represents modes calculated from a sub-sampled set of $\mathbf{X}$. Each sub-sampled set contains the full $12 \mathrm{~s}$. The results in Figure 11 indicate that

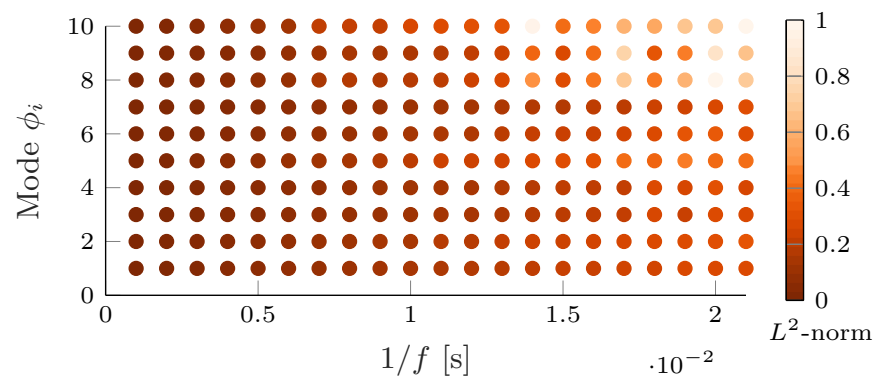

Figure 11: Sampling rate mode convergence for the first ten modes.

a wavenumber of $0.5 \times 10^{-2} \mathrm{~s}$ or, $200 \mathrm{~Hz}$, is a sufficient sampling rate for the most energetic modes. The full sampling rate of $1000 \mathrm{~Hz}$ for $12 \mathrm{~s}$ was used for all investigations since ample storage and computing power was available.

\subsubsection{Two-point correlation}

Two-point correlation is used to evaluate if two spatially separated signals are temporally correlated. The two-point correlation for a flow variable $\xi$ is expressed as

$$
C_{\xi}\left(x_{A}, x_{B}\right)=\overline{\boldsymbol{\xi}^{\prime}\left(x_{A}\right) \boldsymbol{\xi}^{\prime}\left(x_{B}\right)}
$$

where $x_{A}$ and $x_{B}$ are the spatial coordinates of interest and $\boldsymbol{\xi}^{\prime}$ is a vector of the fluctuating flow variable $\xi$. The two point correlation is large and positive for two correlated signals in phase, large and negative for two correlated signals $180^{\circ}$ out of phase and small for uncorrelated signals. A signal correlated with itself is the same as the RMS value of that signal squared. It can be convenient to normalise the two-point correlation to vary between -1 and 1 . The normalised two-point correlation reads

$$
C_{\xi}^{n o r m}\left(x_{A}, x_{B}\right)=\frac{1}{\boldsymbol{\xi}_{R M S}^{\prime}\left(x_{A}\right) \boldsymbol{\xi}_{R M S}^{\prime}\left(x_{B}\right)} \overline{\boldsymbol{\xi}^{\prime}\left(x_{A}\right) \boldsymbol{\xi}^{\prime}\left(x_{B}\right)}
$$

where

$$
\xi_{R M S}^{\prime}(x)=\left(\overline{\xi^{\prime 2}}\right)^{\frac{1}{2}}
$$

Both formulations of the two-point correlation are used in this paper. 


\section{Results and Discussion}

The results for each configuration are presented and discussed in this section, with main focus on the $0^{\circ}$ - and $5.0^{\circ}$-yaw cases. The $0^{\circ}$-yaw flow condition is presented and discussed first followed by $5.0^{\circ}$-yaw.

The lowest drag configuration without side wind is the smooth extensions, $B$, while at yaw the extensions with kick, $C$, provide the lowest drag, seen in Figure 12. This is consistent with previous experimental findings [13]. The extensions with a kick reduce the drag increase seen from yaw, making the drag of the vehicle less sensitive to sidewind.

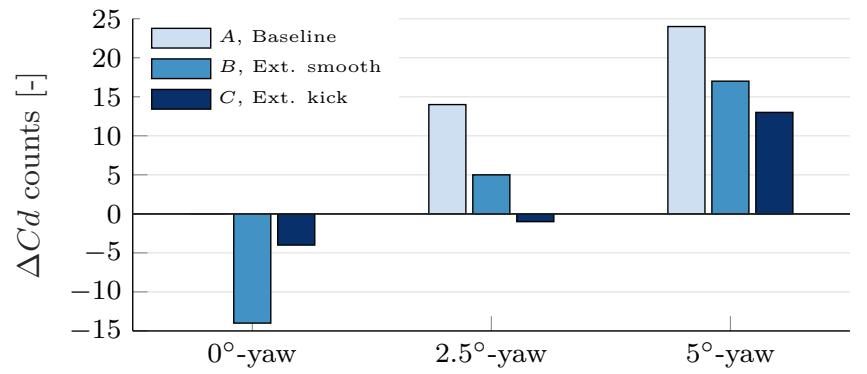

Figure 12: Delta coefficient of drag relative to the baseline

\section{$4.10^{\circ}$-yaw}

As mentioned, Perry [15] did not find a clear connection between drag and the wake closure point, size and the kinetic energy within the wake. However, impingement location and the balance of the recirculating region was reported as important factors linked to drag.

In this study, the wake for configurations $A$ and $C$ have similar shapes with the recirculating flow vectors angled upward, Figure 13. The impingement location is located closer to the top of the vehicle for these configurations. The recirculating flow vectors for configuration $B$ are angled more horizontally and contain larger peak longitudinal velocities. It is believed that the combination of recirculating flow angle and wall normal velocity magnitude is important for the improved base pressure recovery observed on configuration $B$. However, further investigations are needed before the observation can be generalised. The base impingement location is consistent with a region of higher pressure, observed in Figure 13 and 14. For configuration $B$, which has the highest mean base pressure, the flow impingement is located centrally on the base. The base pressures are qualitatively similar for configurations $A$ and $C$ with the largest differences located at the bumper. The bumper for configuration $C$ sees an increased base pressure compared to the baseline, especially on the sides. This is probably due to the extensions shielding the lower part of the base from the wheel wake.

The horizontal distance between the singular points for the time averaged top and bottom shear layers is smaller for configuration $B$. The smaller difference in base pressure on the windscreen and bumper for configuration $B$ is thought to be influenced by this distance.

The extensions were further analysed by comparing the crossflow term in the local drag equation, (4). Figure 15 shows the wake plane $100 \mathrm{~mm}$ behind the vehicle. Qualitatively, the smooth extensions, $B$, has areas of high crossflow which is reduced around the perimeter when adding the kick; however, the baseline configuration has smaller areas of high crossflow compared to both $B$ and $C$. This could be due to the tapering

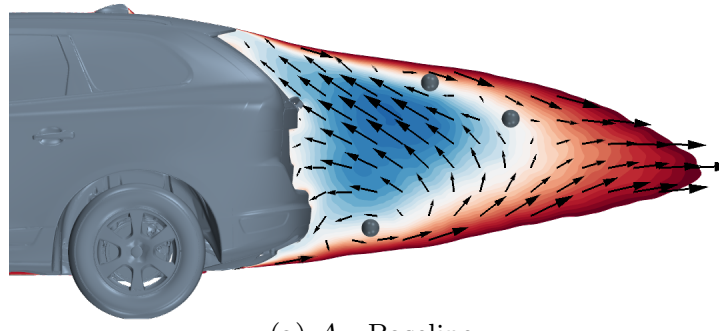

(a) $A$ - Baseline

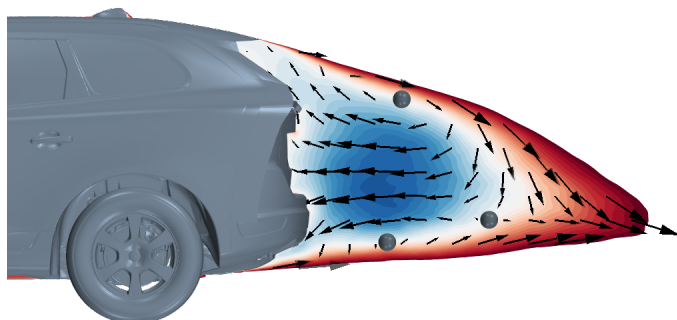

(b) $B$ - Extensions smooth

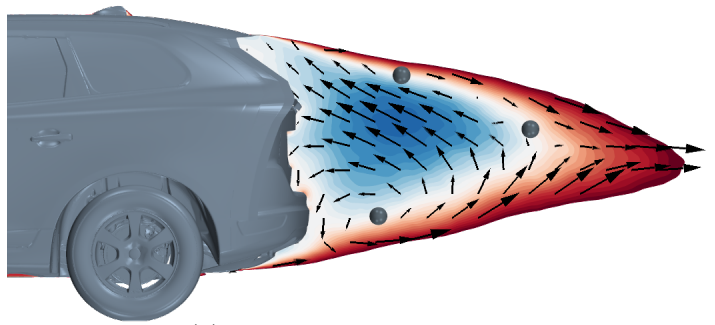

(c) $C$ - Extensions with kick

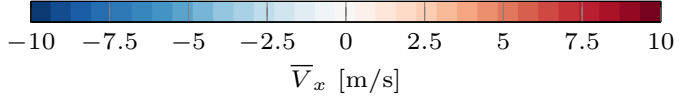

Figure 13: Mean longitudinal velocity with velocity vectors. Time averaged singular points are marked with a sphere.

of the extensions causing higher inflow velocities compared to the baseline.

Since the entire wake plane is included in the local drag equation, the wake plane integral value of crossflow was investigated. This was done in several downstream locations, seen in Figure 16. While the qualitative investigation of the crossflow drag term indicated that the baseline has the lowest crossflow drag, the integral value is in fact larger for the baseline $100 \mathrm{~mm}$ behind the vehicle. The baseline configuration has the largest peak value which is also located closer to the base of the vehicle compared to $B$ and $C$. The peak crossflow drag locations show no correlation with the wake closure points. The near wake and peak crossflow drag value for configuration $C$ is the lowest, indicating that the kick aids in re-aligning the flow to the freestream direction. As it will be shown later, this is beneficial for yawed flow.

This shows that, while the crossflow drag term can give an indication of potential improvement areas in the wake, a qualitative investigation can be misleading when considering the integral value. Note that the local drag equation, eq. (4), can be solved for using only $V_{x}$ and $C_{P}$. The crossflow drag term cancels out if the equation is expanded by substituting the total pressure with static and dynamic pressure, as shown in equation (5).

The RMS pressures were calculated for each configuration to investigate the difference in unsteadiness on the base surface. Physical tests by Duell et al., Sebben et al., Khalighi et al. [3, $13,14]$ have found that the use of base cavities or extensions decrease the base surface pressure fluctuations. Configuration $B$ has the lowest base pressure fluctuations, especially at the center of the base area, which can be seen in Figure 17. The 


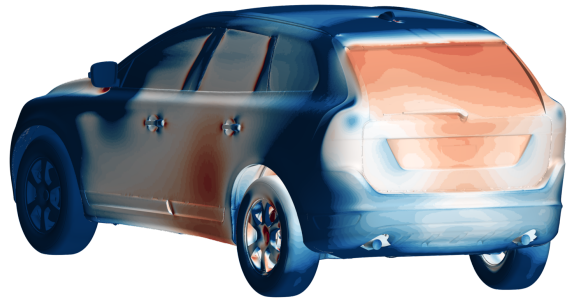

(a) $A$ - Baseline

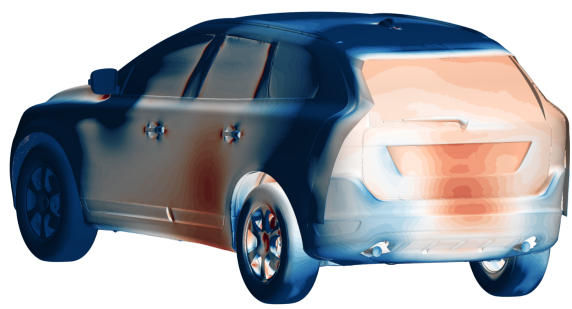

for the full simulation frequency of $4000 \mathrm{~Hz}$ and thus contains fluctuations for a broad range of time scales.

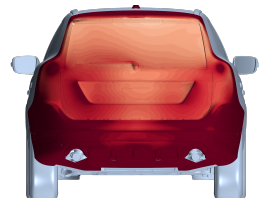

(a) A - Baseline

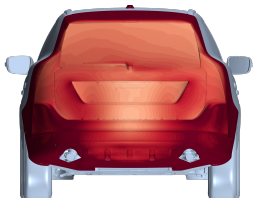

(b) $B$ - Ext. smooth

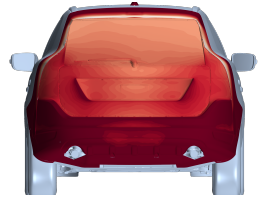

(c) $C$ - Ext. with kick

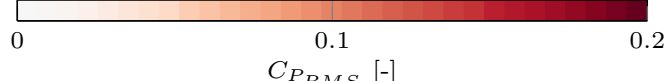

Figure 17: Base RMS pressure (b) $B$ - Extensions smooth

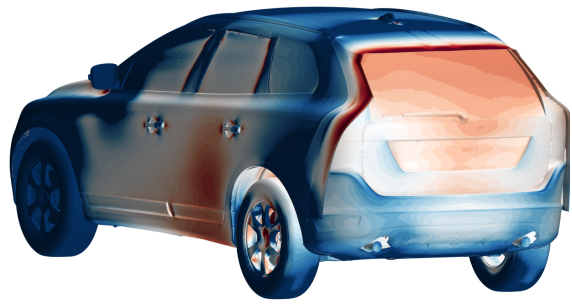

(c) $C$ - Extensions with kick

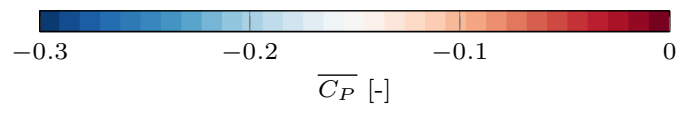

Figure 14: Mean surface pressure coefficient

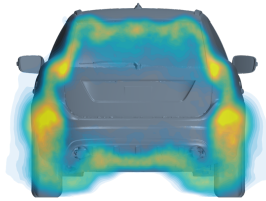

(a) $A$ - Baseline

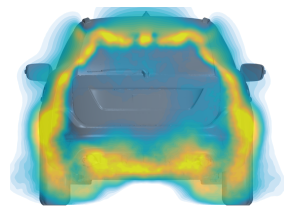

(b) $B$ - Ext. smooth

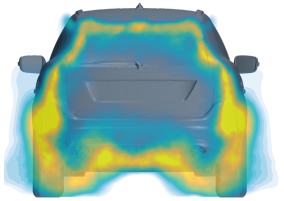

(c) $C$ - Ext. with kick

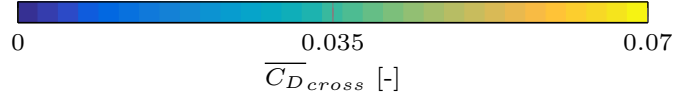

Figure 15: Mean crossflow drag at the $100 \mathrm{~mm}$ plane

extensions with a kick yield local reductions to the RMS base pressure; however, the majority of the surface sees a small increase in base pressure fluctuations compared to the baseline. It should be noted that the RMS base pressure are calculated

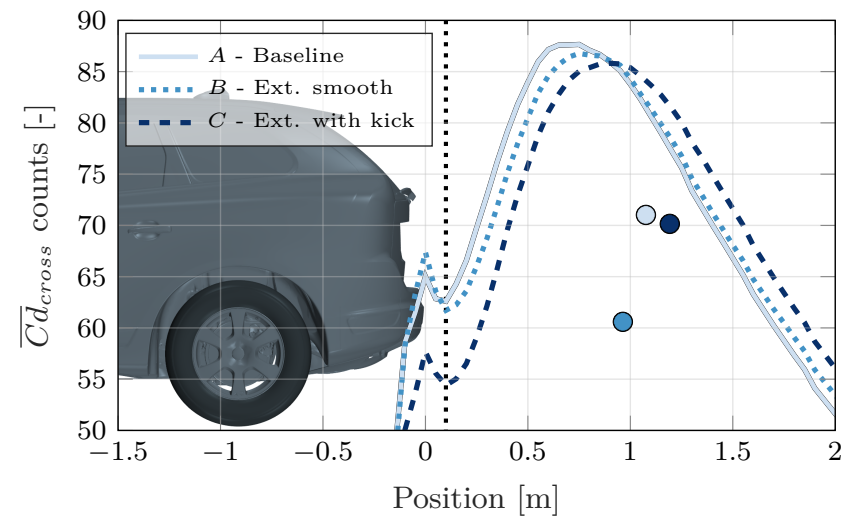

Figure 16: Crossflow drag over X. Marker for wake closure point. Dotted vertical line indicates the $100 \mathrm{~mm}$ location
Two-point correlation was performed for 33 locations distributed across the base where one point was correlated to all other base surface cells. Figure 18 shows the two-point correlation for one of the investigated points, where the correlated reference, $x_{A}$, is illustrated by a yellow sphere. Note that the two-point correlation isn't normalised since the relative magnitudes between configurations is of interest. The magnitude

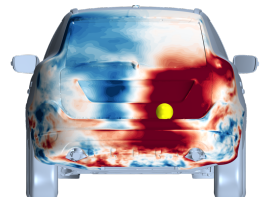

(a) $A$ - Baseline

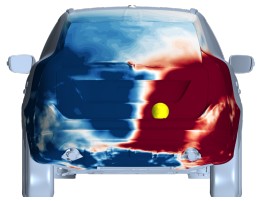

(b) $B$ - Ext. smooth

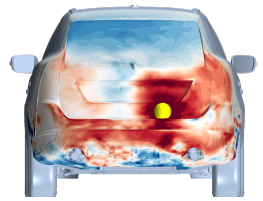

(c) $C$ - Ext. with kick

$$
\begin{array}{ccc}
\hline & & 0 \\
-2.5 & 2.5 \\
& C_{P}^{2}[-] &
\end{array}
$$

Figure 18: Two-point correlation $C_{C_{P}, C_{P}}\left(x_{A}, x_{B}\right)$

of the two-point correlation is large for configuration $B$ with a strong side to side symmetry. This large magnitude of the two-point correlation, together with the low base pressure fluctuations suggest that, even though the fluctuations are weak, the structure is coherent.

Figure 19 shows the averaged Power Spectral Density (PSD) of the 5 largest POD modes where the Strouhal number (St) characteristic length is set to the square root of the frontal area. Configuration $C$ has the smallest peaks and configuration $B$

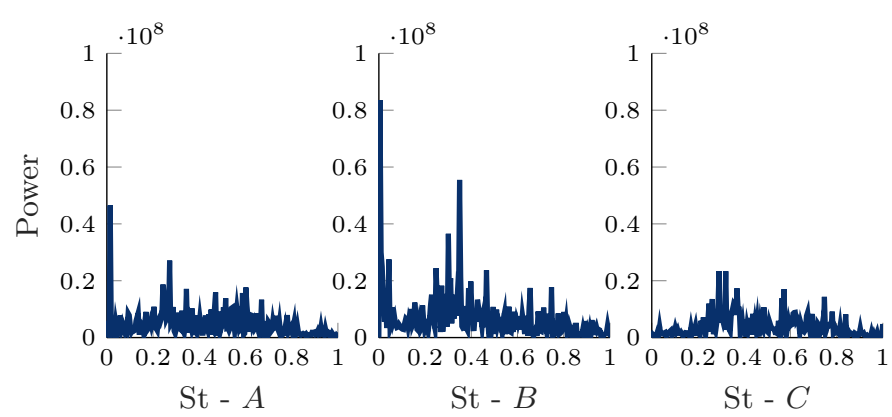

Figure 19: PSD of POD coefficients averaged over 5 largest POD modes

the largest, which is consistent with the relative magnitudes of the two point correlation, Figure 18. This supports the previous theory, that is, the wake of configuration $B$ is comprised of structures that are more coherent than the other configurations. The modal energy, corresponding to $\boldsymbol{\Sigma}$ in equation (11), is plotted in Figure 20 where the modes have been normalised to the largest mode for all data sets. Note that $\boldsymbol{\Sigma}$ is not the kinetic energy since the POD input contains both velocity and pressure. Configuration $C$ has the largest mode energy, for
8 


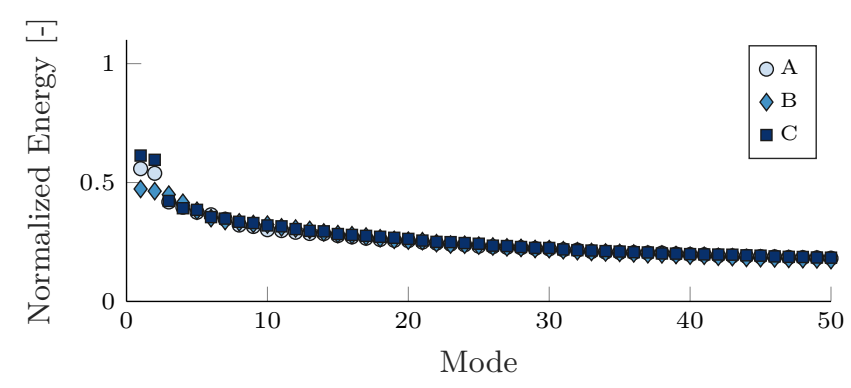

Figure 20: Normalised energy of 50 largest modes

a given mode, and corresponds to a mode located in the top shear layer. This mode is shown in Figure 21 and has it's peak
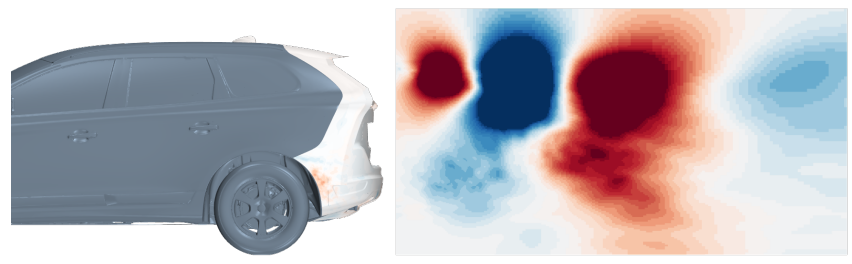

Figure 21: Configuration $C$. First POD mode, side view

frequency at $S t=0.29$. The first and second modes for configuration $C$ are both top shear layer modes with frequency peaks located at $S t=0.29$, the fourth and fifth modes are also located in the top shear layer with peaks at $S t=0.57$. The respective frequency peaks can be seen in Figure 19 which, as previously mentioned, contains the average PSD of the five largest modes. Figure 22 shows the fourth mode for configuration $C$ and, due
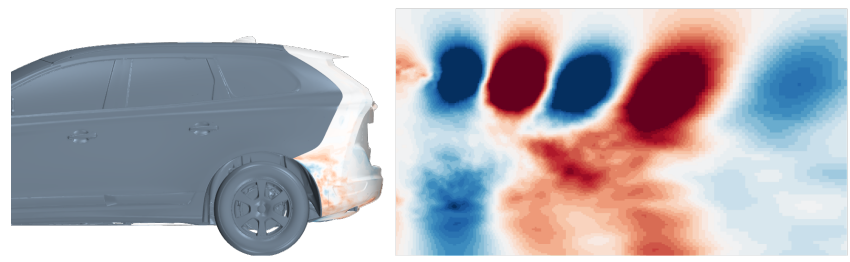

Figure 22: Configuration $C$. Fourth POD mode, side view

to the location and frequency of the mode, is thought to be part of the same structure as found in the first and second modes. The shear layer modes are found in configurations $A$ and $B$ as well; however, the energy and relative positions vary. For configuration $B$ the shear layer mode is strong in both the top and bottom, this suggests that the large energy containing fluctuations in the top and bottom shear layers are of similar importance.

The first, second and forth modes for configuration $B$ all show a strong side to side dependency. The forth mode for configuration $B$ is illustrated in Figure 23 where the side to side antisymmetry can be seen. For configuration $B$ the first, second and fourth mode have peaks at Strouhal numbers 0.35, 0.3, 0.40 respectively. Additionally a low frequency peak at $S t \approx 0.1$ is found for each side to side anti-symmetric mode. These low frequencies have typically been associated with a pumping motion [13]; however, here no connection was found at $0^{\circ}$-yaw for the first ten modes. A pumping mode is observed at $5^{\circ}$-yaw for configuration $A$. Configuration $B$ contains energetic modes with side to side anti-symmetry, which is consistent with the previously presented two-point correlation results, Figure 18. The frequencies at which the anti-symmetric modes were found varied for each configuration and also between modes, but were found in the range $S t=0.3-0.6$.

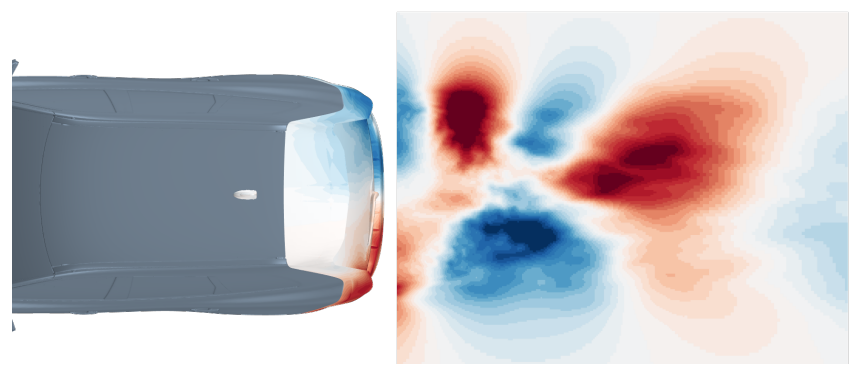

Figure 23: Configuration $B$. Fourth POD mode, top view

One of the drawbacks of Proper Orthogonal Decomposition is that each mode can contain a broad range of frequencies, an alternative method which extracts modes containing one frequency is Dynamic Mode Decomposition; however, selecting which modes are of high importance to the data-set is difficult [30]. The kick reduces the energy in the anti-symmetric modes, with the first anti-symmetric mode found at POD mode number seven.

Consistent conclusions could not be drawn from the modal analysis to it's influence on drag. However, the following observations could be made: (i) Strong modes with large peak fluctuations is not an indication of poor aerodynamic performance alone; in fact, configuration $B$ has the largest modal peaks in the Power Spectral Diagram as well as several anti-symmetric modes, yet, has the lowest drag. (ii) The modal results suggest that configuration $B$ could benefit from hindering the side to side interaction, and more so than the other configurations. The anti-symmetric or side to side wake movement has been linked to drag, Ahmed et al. [25] showed that the drag on a bluff body could be reduced by placing a vertical splitter plate along the symmetry line to hinder the side to side interaction in the wake.

\section{2 $5.0^{\circ}$-yaw}

The results for the vehicle at $5.0^{\circ}$-yaw are presented in this section. In each figure, the windward side is on the left side of the vehicle in the driving direction. In other words, the side wind is moving from left to right in Figure 24. The local drag $100 \mathrm{~mm}$ behind the vehicle reveals that the recirculating flow for configuration $C$ is located centrally at the base, Figure 24 . Configuration $A$ and $B$ have similar wake structures although

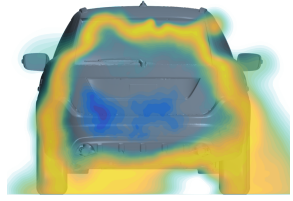

(a) A - Baseline

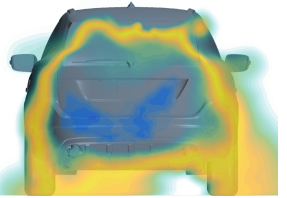

(b) $B$ - Ext. smooth (c) $C$ - Ext. with kick

$\begin{array}{llllll}-0.3 & -0.1 & 0.1 & 0.3 & 0.5 & 0.7 \\ & & \bar{C}_{\text {Dlocal }}[-] & & \end{array}$

Figure 24: Local drag

the perimeter of high losses has spread more for configuration $A$. This could in large part be due to the flow separating earlier for configuration $A$ than $B$.

Since the vehicle isn't subjected to aerodynamically symmetrical conditions it is not fair to investigate the centerline wake structures between configurations, as it was done for $0^{\circ}$-yaw. Instead, a top view is used for the yawed configurations. The plane in Figure 25 is positioned in the area of high negative local drag for configuration $A$ and $B$, Figure 24, which is located 


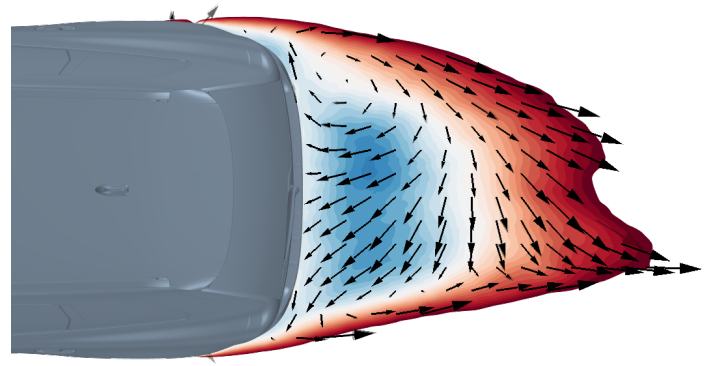

(a) A - Baseline

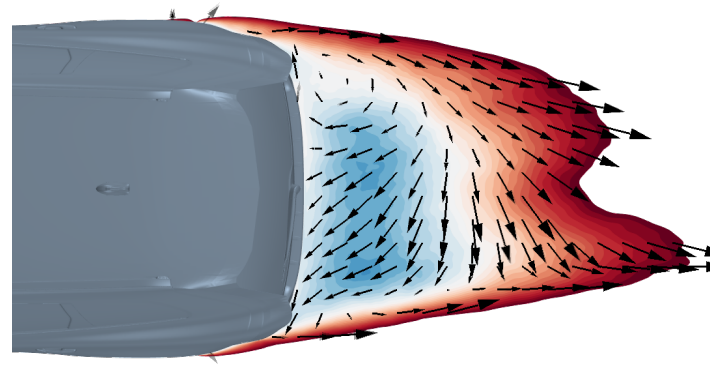

(b) $B$ - Extensions smooth

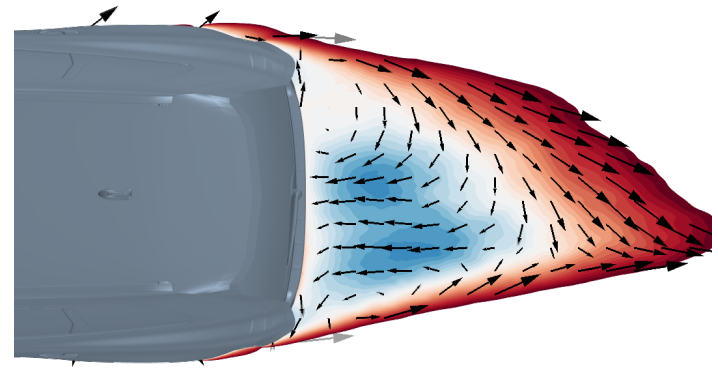

(c) $C$ - Extensions with kick

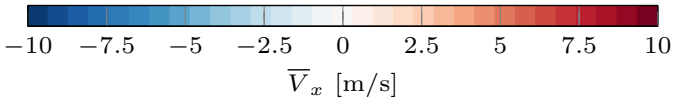

Figure 25: Mean longitudinal velocity, top approximately $0.4 H$ from the ground with $H$ being the total vehicle height. The lowest drag configuration at yaw, configuration $C$, is characterised by perpendicular recirculating flow, which can be seen in Figure 25 .

Figure 26 shows an improved pressure recovery for the extensions with a kick, especially at the windward side. The high pressure location is consistent with the negative local drag for each configuration.

Figure 27 shows the crossflow drag overlain with velocity vectors for the wake plane $500 \mathrm{~mm}$ behind the vehicle. A large scale twisting motion of the wake is present for configurations $A$ and $B$ which is also seen for $2.5^{\circ}$-yaw. The kick on the extensions aid in reducing the twisting wake structure, resulting in an improved wake structure and lower drag. For configuration $C$, the large rotation is replaced by two smaller rotating structures which moves the base impinging positing upward and closer to the centerline. This is illustrated schematically in Figure 28 for configurations $B$ and $C$. It is believed that the large scale rotation is hindering base pressure recovery while also consum-

Time dependent post-processing was also done for the yawed flow condition revealing similar trends as for $0^{\circ}$-yaw, albeit differing between configurations. The RMS base pressure fluctuations are reduced for configuration $C$ on the windward side and close to the impingement location of the recirculating flow compared to configurations $A$ and $B$. ing energy.

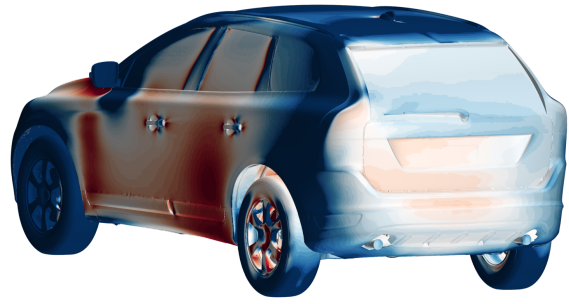

(a) $A$ - Baseline

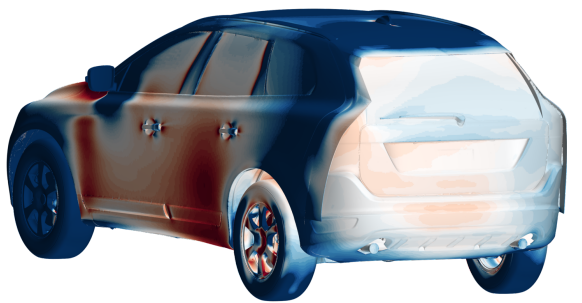

(b) $B$ - Extensions smooth

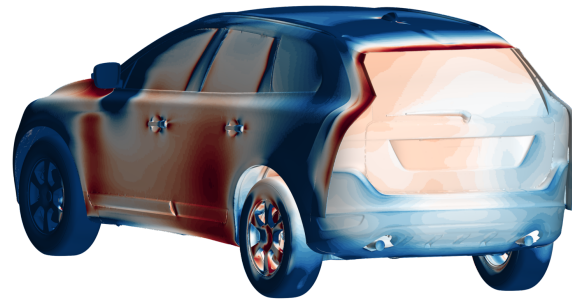

(c) $C$ - Extensions with kick

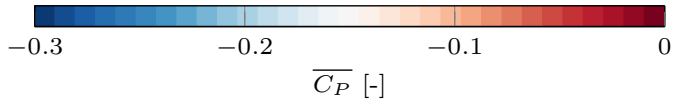

Figure 26: Mean surface pressure coefficient

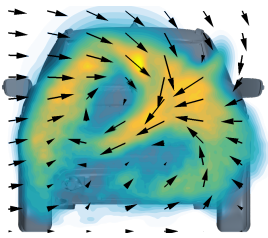

(a) $A$ - Baseline

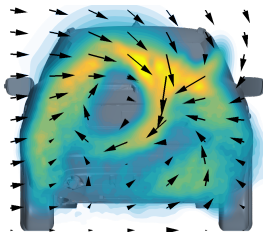

(b) $B$ - Ext. smooth

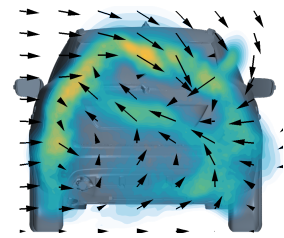

(c) $C$ - Ext. with kick
Figure 27: Mean crossflow drag with velocity vectors, $500 \mathrm{~mm}$ behind the vehicle

However, the two-point correlation at yaw did not reveal the same strong symmetry as previously seen at $0^{\circ}$-yaw, for any of the 33 investigated points. The two-point correlation does, however, show a larger positively correlated area for all configurations. The correlation is weakened by the extensions, especially when combined with the kick, Figure 29. The surface base RMS pressures coupled with the two-point correlation indicate that the near wake large scale turbulent motion is reduced when
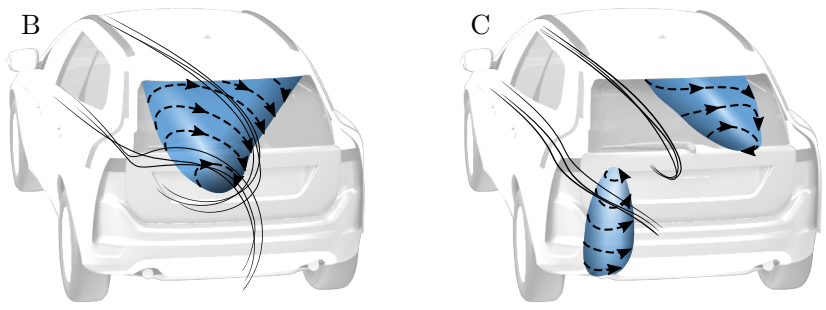

Figure 28: Schematic overview of the wake twisting 
using extensions at yaw.

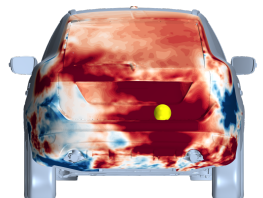

(a) A - Baseline

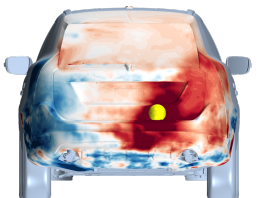

(b) $B$ - Ext. smooth

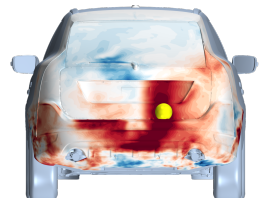

(c) $C$ - Ext. with kick

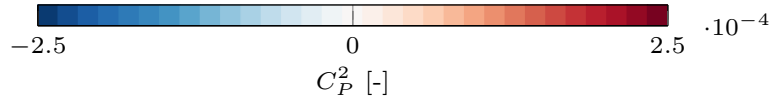

Figure 29: Two-point correlation $C_{C_{P}, C_{P}}\left(x_{A}, x_{B}\right)$

At yaw, the shear layer mode is shifted to one side of the vehicle. Figure 30 shows the shear layer mode, mode one, for configuration $A$ from the top where the shear layer mode on the windward side can be seen. This is similar for the other configurations. The anti-symmetric modes are not as pronounced in the yawed case and are only seen in the higher mode numbers, above five.
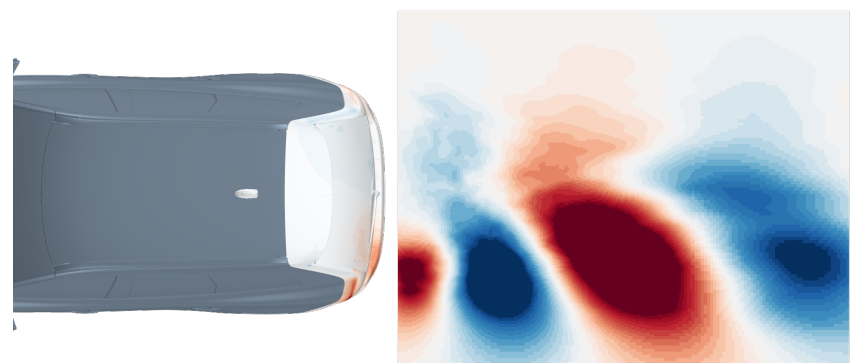

Figure 30: Configuration $A$. First POD mode, top view

At $0^{\circ}$-yaw each anti-symmetric mode was accompanied with a low frequency peak. A low frequency peak has previously been linked to a pumping mode [13], this modal shape was not found for the first ten modes at $0^{\circ}$-yaw. At yaw, the fifth mode for the configuration $A$ indicates such a pumping mode with a peak frequency in the PSD at $S t=0.01$. The pumping mode is shown on the centerline, y-normal plane, in Figure 31 where a large positively correlated area can be seen on the top part of the wake. The low frequency peak can be seen in the average of the first five modes, Figure 32. The pumping mode was not found in the first ten modes for the other configurations at yaw. It should be noted that the current modal investigation was

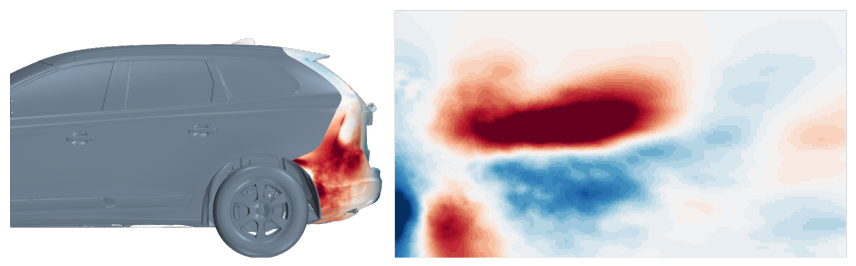

Figure 31: Configuration $A$. Fifth POD mode. Pumping mode, side view

done on the vehicle base and in three planes which means that the extracted modes and relative modal energy could change if a larger volume was included. The normalised energy for the largest modes is larger when at yaw which can be seen by comparing Figure 20 and 33. It's interesting to note that the available kinetic energy in the freestream is the same for both flow conditions, it's only the incoming flow angle which differs. The modal energy is especially large for the high energy modes associated with the windward shear layer when at yaw.

The peak frequency of the shear layer modes was reduced when adding a kick, compared to smooth extensions or no extensions.

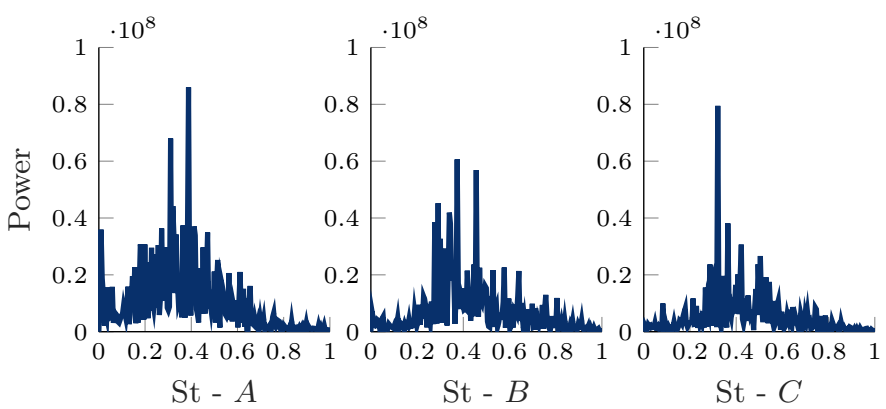

Figure 32: PSD of POD coefficients averaged over 10 largest POD modes

The peak frequency of the shear layer modes was reduced from Strouhal number 0.38 to 0.32 for when adding the kick.

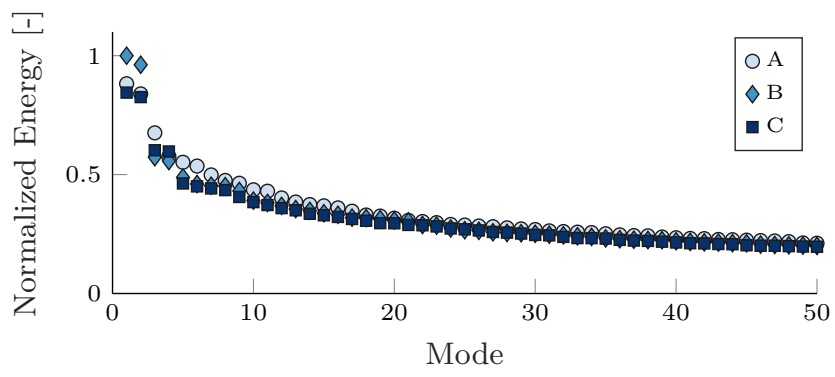

Figure 33: Normalised energy of 50 largest modes

\section{Concluding remarks}

Tapered rear end extensions with and without a kick were numerically investigated for straight and yawed flow conditions, representing a constant side wind. The main conclusions of this study are presented next.

- Wall normal recirculating flow angle and large negative longitudinal velocity were found for the lowest drag configurations with and without side wind.

- The base pressure recovery is improved when using extensions with a kick at yaw, especially on the windward side.

- The extensions reduce the RMS pressure fluctuations at the impingement location of the recirculating flow.

- Extensions with a kick reduce a large scale twisting motion present in the baseline and smooth extensions for the investigated $2.5^{\circ}$ - and $5^{\circ}$-yaw.

- Modal analysis revealed strong anti-symmetric modes for the smooth extensions at $0^{\circ}$-yaw. The modes could not be linked to drag alone; however, indications of potential improvement areas were found.

- The peak frequency of the shear layer modes was reduced from Strouhal number 0.38 to 0.32 when adding a kick compared to smooth extensions or no extensions.

- The modal energy in the POD modes increased when at yaw, despite the available kinetic energy being the same.

- The anti-symmetric modes were reduced at yaw for all configurations.

Further, the two-point correlation combined with RMS base pressure indicate that the smooth extensions in straight flow reduce the near wake turbulence as well as contribute to a coherent side to side motion. At yaw, the coherent motion is 
not as pronounced; however, the correlated areas are reduced when using extensions, suggesting a reduction in the near wake turbulent structures.

The crossflow drag term in the local drag equation, eq. (4), can give an indication of potential improvement areas as was seen for the $5^{\circ}$-yaw case. However, misleading results were observed when comparing qualitative and integral results at $0^{\circ}$-yaw. It is recommended that the local drag equation, eq. (4), be solved for in its expanded form, eq. (5), i.e. without the $V_{y}$ and $V_{z}$ components. The crossflow drag term could be regarded as a quantity separate from local drag.

\section{Acknowledgements}

The authors would like to thank Alexander Broniewicz and Volvo Car Corporation for their support and cooperation, without which this work would not have been possible. The simulations and computationally heavy post-processing was performed on resources provided by the Swedish National Infrastructure for Computing (SNIC) at PDC. [32]

\section{Funding}

This work is funded by the Swedish Energy Agency P43328-1. 


\section{References}

[1] Wolf-Heinrich Hucho, ed. Aerodynamics of Road Vehicles: From Fluid Mechanics to Vehicle Engineering. 4 edition. Warrendale, PA: Society of Automotive Engineers Inc, Feb. 1998. 918 pp. ISBN: 978-0-7680-0029-0.

[2] Felix Wittmeier and Timo Kuthada. "The influence of wheel and tire aerodynamics in WLTP". In: 6th International Munich Chassis Symposium 2015. Ed. by Peter Pfeffer. Wiesbaden: Springer Fachmedien Wiesbaden, 2015, pp. 149-160.

[3] Edward G. Duell and A. R. George. Experimental study of a ground vehicle body unsteady near wake. SAE Technical Paper, 1999.

[4] Jeff Howell et al. "Bluff Body Drag Reduction with Ventilated Base Cavities". In: SAE International Journal of Passenger Cars - Mechanical Systems 5.1 (Apr. 16, 2012), pp. 152-160. DOI: $10.4271 / 2012-01-0171$.

[5] Kevin R. Cooper. "Truck Aerodynamics Reborn - Lessons from the Past". In: Nov. 10, 2003. DOI: $10.4271 / 2003-$ 01-3376.

[6] YA Irving Brown, S. Windsor, and A. P. Gaylard. The effect of base bleed and rear cavities on the drag of an SUV. SAE Technical Paper, 2010.

[7] Anna-Kristina Perry, Giancarlo Pavia, and Martin Passmore. "Influence of short rear end tapers on the wake of a simplified square-back vehicle: wake topology and rear drag". In: Experiments in Fluids 57.11 (Nov. 1, 2016), p. 169. DOI: $10.1007 / \mathrm{s} 00348-016-2260-3$.

[8] Jean-Luc Aider, Jean-François Beaudoin, and José Eduardo Wesfreid. "Drag and lift reduction of a 3D bluffbody using active vortex generators". en. In: Experiments in Fluids 48.5 (May 2010), pp. 771-789. DOI: 10.1007/ s00348-009-0770-y.

[9] Jonathan McNally et al. "Drag reduction on a flat-back ground vehicle with active flow control". In: Journal of Wind Engineering and Industrial Aerodynamics 145 (Oct. 1, 2015), pp. 292-303. DOI: 10 . 1016/j · jweia . 2015.03 .006 .

[10] Azeddine Kourta and Cédric Leclerc. "Characterization of synthetic jet actuation with application to Ahmed body wake". en. In: Sensors and Actuators A: Physical 192 (Apr. 2013), pp. 13-26. DOI: 10.1016/j.sna.2012.12. 008.

[11] Rustan Tarakka, Sabar P. Simanungkalit, et al. "Effect of active control by blowing to aerodynamic drag of bluff body van model". In: International Journal of Fluid Mechanics Research 40.4 (2013).

[12] Bae Geun Hwang et al. "Reduction of drag in heavy vehicles with two different types of advanced side skirts". In: Journal of Wind Engineering and Industrial Aerodynamics 155 (Aug. 1, 2016), pp. 36-46. DOI: 10.1016/j . jweia.2016.04.009.

[13] Simone Sebben, Lennert Sterken, and Thies Wölken. "Characterization of the rear wake of a sport utility vehicle with extensions and without extensions". In: Institution of Mechanical Engineers, Part D: Journal of Automobile Engineering (2016). DOI: DOI : 10 . 1177 / 0954407016678016.
[14] Bahram Khalighi, Szabolcs R. Balkanyi, and Luis P. Bernal. "Experimental investigation of aerodynamic flow over a bluff body in ground proximity with drag reduction devices". In: International Journal of Aerodynamics 3.4 (2013), p. 217. DOI: 10.1504/IJAD. 2013.054421.

[15] Anna K. Perry. "An investigation into the base pressure of simplified automotive squareback geometries". PhD thesis. Loughborough University, 2016.

[16] Kunihiko Taira et al. "Modal Analysis of Fluid Flows: An Overview". In: arXiv:1702.01453 [physics] (Feb. 5, 2017). arXiv: 1702.01453.

[17] Lennert Sterken et al. "Effect of Rear-End Extensions on the Aerodynamic Forces of an SUV". In: Apr. 1, 2014. DOI: $10.4271 / 2014-01-0602$.

[18] Lennert Sterken et al. "Wake and Unsteady SurfacePressure Measurements on an SUV with Rear-End Extensions". In: Apr. 14, 2015. DOI: 10.4271/2015-011545.

[19] Teddy Hobeika and Simone Sebben. "CFD investigation on wheel rotation modelling". In: Journal of Wind Engineering and Industrial Aerodynamics 174 (Mar. 2018), pp. 241-251. DOI: $10.1016 / \mathrm{j}$. jweia.2018.01.005.

[20] Lars Davidson. "Large eddy simulations: how to evaluate resolution". In: International Journal of Heat and Fluid Flow (2009).

[21] Grégoire Fourrié et al. "Bluff-body drag reduction using a deflector". In: Experiments in Fluids 50.2 (Feb. 2011), pp. 385-395. DOI: $10.1007 / \mathrm{s} 00348-010-0937-6$.

[22] E Maskell. Progress towards a method for the measurement of the components of the drag of a wing of finite span. 1973.

[23] J. C. Wu. A Generalized Wake-Integral Approach for Drag Determination in Three-Dimensional Flows. 1979.

[24] G. Mccutcheon et al. "Wake Studies of a Model Passenger Car Using PIV". In: Dec. 2, 2002. DOI: 10.4271/200201-3335.

[25] S. R. Ahmed. "Wake structure of typical automobile shapes". In: ASME J. Fluids Eng 103 (1981), pp. 162169 .

[26] M. Onorato, A.F. Costelli, and A. Garrone. "Drag Measurement Through Wake Analysis". In: Feb. 1, 1984. DOI: $10.4271 / 840302$.

[27] Antonello Cogotti. A Strategy for Optimum Surveys of Passenger-Car Flow Fields. SAE Technical Paper, 1989.

[28] P. W. Bearman. Some observations on road vehicle wakes. SAE Technical Paper, 1984.

[29] Alberto Morelli. A New Aerodynamic Approach to Advanced Automobile Basic Shapes. 2000.

[30] Tomas W. Muld, Gunilla Efraimsson, and Dan S. Henningson. "Mode Decomposition on Surface-Mounted Cube". In: Flow, Turbulence and Combustion 88.3 (Apr. 2012), pp. 279-310. DOI: 10.1007/s10494-011-9355-y.

[31] S. R. Ahmed, G. Ramm, and G. Faltin. Some salient features of the time-averaged ground vehicle wake. SAE Technical Paper, 1984.

[32] Swedish National Infrastrucutre for Computing. URL: http://www.snic.vr.se/. 